\title{
Iterative robust downlink beamforming in cognitive radio networks
}

\author{
Imran Wajid Hristina Nikolaeva Marius Pesavento \\ Technische Universität Darmstadt, D-64283, Darmstadt, Germany
}

\begin{abstract}
The problem of multi-user worst-case robust downlink beamforming and power allocation in a cognitive radio (CR) framework is studied. The goal is to minimize the total transmitted power while providing the secondary users (SUs) with a minimum acceptable quality-of-service $(\mathrm{QoS})$, with additional constraints on the total interference leaked to the primary network (PN). An iterative algorithm has been proposed to solve this problem. In each iteration the algorithm approximates the worst-case covariance mismatch from the solution of the previous iteration und performs a fixed-point iteration that is based on uplink-downlink duality. Computer simulations show that the proposed technique converges to the global optimal solution at reduced computational complexity compared to the existing methods that rely on convex optimization.
\end{abstract}

Index Terms-cognitive radio, robustness, iterative methods, user quality-of-service

\section{INTRODUCTION}

Current spectrum licensing policies employ fixed spectrum assignment in general. However, since the bandwidth demand by the users varies with time, the spectrum is generally not completely utilized. The underutilization of radio spectrum has been reported, for example, in [1], [2] and [3]. It is therefore natural to seek for a more effective way of exploiting the unused bandwidth. The concept of cognitive radio (CR) has emerged as a promising solution for the efficient utilization of the radio spectrum [4]. In a CR network, the secondary network ( $\mathrm{SN}$ ) is granted access to the licensed spectrum of the primary network $(\mathrm{PN})$ under the premise that the transmissions in the SN do not adversely affect the operation of the PN. In this regard, controlling the interference caused to the PN, due to the transmissions in the $\mathrm{SN}$, is an essential system requirement. A practical scenario to which the concept of CR networks applies, is the deployment and the operation of femto- and picocells within a cellular macro-network. In femtocells a home base station opportunistically uses the spectrum of the surrounding macro network [5]. Downlink beamforming and power allocation techniques can be employed at the SN base station for the efficient control of the interference to the PN while maintaining a minimum required signal-tointerference-plus-noise-ratio (SINR) for the secondary users (SUs), [6]-[11].

Several CR downlink beamforming techniques have been developed which heavily rely on the perfect knowledge of channel state information (CSI) at the SN base station, [6][8]. In practice, however, the availability of error-free CSI is usually not possible. The errors are inevitable in the CSI estimation process and the feedback procedure, [12] and [13].
The methods based on perfect CSI are quite sensitive to the mismatches in CSI, which motivates the development of techniques that are robust to these CSI errors. Several robust downlink beamforming techniques have recently been proposed [9]-[11], among which the method of [9] is suboptimal in the sense that it involves several conservative approximations. The approaches in [10] and [11] reformulate the corresponding robust problems exactly, however, under certain conditions, the scheme in [11] yields higher rank solutions [14], and finding a rank-one feasible point in this case may not be possible. In addition, all these techniques require to solve semidefinite programming (SDP) problems, that involve the use of interior-point algorithms. This makes all these approaches computationally complex and, therefore, not favorable to be implemented on real hardware or DSP architectures.

In this paper, we consider the problem of optimum power allocation and downlink beamforming in a SN. Our aim is to minimize the total transmitted power while providing the SUs with a minimum acceptable quality-of-service (QoS). Additionally, the SN base station must also meet the interference constraints imposed by the PN. In the proposed approach, we combine the benefits of the methods presented in [7] and [11]. In [7], the uplink-downlink duality has been proven for nonrobust CR beamforming problem and an iterative algorithm has been proposed to obtain the optimal beamformers and power allocations for the CR downlink beamforming problem. The algorithm is computationally efficient and simple to implement. In our work, the iterative scheme originally developed for the non-robust problem is adapted to work in case of the robust problem formulation of [11]. The proposed scheme retains the benefit of computational efficiency and shows a convergence rate similar to the non-robust iterative technique. Another advantage of the proposed algorithm is that, when feasible, it always obtains rank-one solutions. Simulation results show that in the case that the method of [11] yields an optimal solution (i.e. a rank-one) then this solution is also attained by the proposed algorithm.

\section{Problem Formulation}

Let us consider a CR network with $L$ primary users (PUs) and $K$ SUs. The SN base station contains $N$ transmit antennas. The receiver of each PU and SU is assumed to consist of a single receive antenna. Without any loss of generality, the SUs are indexed from $1, \ldots, K$, while the PUs are indexed from $K+1, \ldots, K+L$. At time instance $t$, the $\mathrm{SN}$ base station 
transmits the $N \times 1$ vector

$$
\boldsymbol{x}(t)=\sum_{i=1}^{K} \boldsymbol{w}_{i} s_{i}(t)=\boldsymbol{U} \operatorname{diag}\left\{\sqrt{p_{1}}, \ldots, \sqrt{p_{K}}\right\} \boldsymbol{s}(t)
$$

where $s(t) \triangleq\left[s_{1}(t), \ldots, s_{K}(t)\right]^{T}, s_{i}(t)$ is the signal intended for the $i$ th $\mathrm{SU}, \boldsymbol{w}_{i} \triangleq \sqrt{p_{i}} \boldsymbol{u}_{i}, \boldsymbol{U} \triangleq\left[\boldsymbol{u}_{1}, \ldots, \boldsymbol{u}_{K}\right], p_{i}$ denotes the transmitted power allocated to the $i$ th $\mathrm{SU}, \boldsymbol{u}_{i}$ denotes the $N \times 1$ normalized beamforming vector of the $i$ th $\mathrm{SU}, \operatorname{diag}\{\cdot\}$ denotes a diagonal matrix, and $(\cdot)^{T}$ stands for the transpose. The signal received by the $k$ th $\mathrm{SU}$ is given by

$$
y_{k}(t)=\boldsymbol{h}_{k}^{H} \boldsymbol{x}(t)+n_{k}(t)
$$

where $\boldsymbol{h}_{k}$ is the $N \times 1$ vector containing the flat-fading channel gains between the $\mathrm{SN}$ base station and the $k$ th $\mathrm{SU}$ receiver, $n_{k}(t)$ is the noise of $k$ th SU, and $(\cdot)^{H}$ denotes the Hermitian transpose. The receiver noise is assumed to be zero-mean circularly symmetric white Gaussian with variance $\sigma_{k}^{2}$ and independent among users. The SINR at the $k$ th SU receiver can be expressed as [15]

$$
\operatorname{SINR}_{k}=\frac{p_{k} \boldsymbol{u}_{k}^{H} \boldsymbol{R}_{k} \boldsymbol{u}_{k}}{\sum_{\substack{i=1 \\ i \neq k}}^{K} p_{i} \boldsymbol{u}_{i}^{H} \boldsymbol{R}_{k} \boldsymbol{u}_{i}+\sigma_{k}^{2}}
$$

where $\boldsymbol{R}_{k} \triangleq \mathrm{E}\left\{\boldsymbol{h}_{k} \boldsymbol{h}_{k}^{H}\right\}$ is the downlink channel covariance matrix for the $k$ th $\mathrm{SU}$ and $\mathrm{E}\{\cdot\}$ denotes the statistical expectation. The interference power at the $l$ th PU caused by the transmissions from the SN base station can be written as

$$
\mathcal{I}_{l}=\sum_{i=1}^{K} p_{i} \boldsymbol{u}_{i}^{H} \boldsymbol{R}_{K+l} \boldsymbol{u}_{i}
$$

where $\boldsymbol{R}_{K+l} \triangleq \mathrm{E}\left\{\boldsymbol{h}_{K+l} \boldsymbol{h}_{K+l}^{H}\right\}$ is the channel covariance matrix between the SN base station and the $l$ th PU, and $\boldsymbol{h}_{K+l}$ is the corresponding $N \times 1$ channel vector.

In the CR framework, with the assumption of the perfect knowledge of the channel covariance matrices $\boldsymbol{R}_{1}, \ldots, \boldsymbol{R}_{K+L}$, we design the beamforming matrix $\boldsymbol{U}$ and the downlink powers $p_{1}, \ldots, p_{K}$ to minimize the total transmitted power $\sum_{i=1}^{K} p_{i}$ subject to the minimum QoS requirements for each $\mathrm{SU}$ and with the interference at the individual PUs below a certain predefined interference temperature. The resulting beamforming problem can be written as

$$
\begin{array}{ll} 
& \min _{\left\{\boldsymbol{U}, p_{1}, \ldots, p_{K}\right\}} \sum_{i=1}^{K} p_{k} \\
\text { s.t. } & \frac{p_{k} \boldsymbol{u}_{k}^{H} \boldsymbol{R}_{k} \boldsymbol{u}_{k}}{\sum_{\substack{i=1 \\
i \neq k}}^{K} p_{i} \boldsymbol{u}_{i}^{H} \boldsymbol{R}_{k} \boldsymbol{u}_{i}+\sigma_{k}^{2}} \geq \gamma_{k} ; k=1, \ldots, K \\
& \sum_{i=1}^{K} p_{i} \boldsymbol{u}_{i}^{H} \boldsymbol{R}_{K+l} \boldsymbol{u}_{i} \leq \frac{1}{\gamma_{K+l}} ; l=1, \ldots, L
\end{array}
$$

where $\gamma_{k}$ is the minimal acceptable SINR of the $k$ th $\mathrm{SU}, \frac{1}{\gamma_{K+l}}$ is the upper limit on the interference power caused by the $\mathrm{SN}$ base station at the $l$ th $\mathrm{PU}$, and $\|\cdot\|$ denotes the Frobenius norm of a matrix or the Euclidean norm of a vector.

The non-robust CR downlink beamforming problem (5) has previously been addressed in [7] and [9] and can be viewed as an extension to the conventional downlink beamforming problem of [15] with added PU interference constraints. In [9], new matrix variables, defined as $\boldsymbol{W}_{k} \triangleq p_{k} \boldsymbol{u}_{k} \boldsymbol{u}_{k}^{H}$, are introduced and the problem (5) is reformulted as

$$
\begin{array}{ll} 
& \min _{\left\{\boldsymbol{W}_{i}\right\}} \sum_{i=1}^{K} \operatorname{Tr}\left\{\boldsymbol{W}_{i}\right\} \\
\text { s.t. } & \operatorname{Tr}\left\{\boldsymbol{W}_{k} \boldsymbol{R}_{k}\right\}-\gamma_{k} \sum_{\substack{i=1 \\
i \neq k}}^{K} \operatorname{Tr}\left\{\boldsymbol{W}_{i} \boldsymbol{R}_{k}\right\} \geq \eta_{k} \\
& \boldsymbol{W}_{k} \geq 0, \operatorname{rank}\left(\boldsymbol{W}_{k}\right)=1 ; k=1, \ldots, K \\
& -\gamma_{K+l} \sum_{i=1}^{K} \operatorname{Tr}\left\{\boldsymbol{W}_{i} \hat{\boldsymbol{R}}_{K+l}\right\} \geq-1 ; l=1, \ldots, L
\end{array}
$$

where $\eta_{k} \triangleq \sigma_{k}^{2} \gamma_{k}$ and $\operatorname{Tr}\{\cdot\}$ denotes the trace of a matrix. Next, following the semidefinite relaxation (SDR) approach, the nonconvex rank-one constraint is dropped to convert the problem (6) into a convex form. It has been shown in [7] and [16], for CR and conventional downlink beamforming, respectively, that the SDR step does not involve any approximation.

In [7], a dual virtual uplink problem corresponding to the problem (5) is derived as

$$
\begin{array}{ll} 
& \min _{\substack{\left\{, q_{1}, \ldots, q_{K+L}\right\} \\
i=1}} \sum_{i=1}^{K} \eta_{k} q_{k}-\sum_{l=1}^{L} p_{K+L} q_{K+L} \\
\text { s.t. } & \frac{q_{k} \boldsymbol{u}_{k}^{H} \boldsymbol{R}_{k} \boldsymbol{u}_{k}}{} \\
& \boldsymbol{u}_{k}^{H}\left(\sum_{\substack{i=1 \\
i \neq k}}^{K+L} q_{i} \gamma_{i} \boldsymbol{R}_{i}+\boldsymbol{I}\right) \boldsymbol{u}_{k} \\
& q_{k} \geq 0 ; q_{K+l} \geq 0 ; p_{K+l} \leq 1 ;\left\|\boldsymbol{u}_{k}\right\|=1 ; l=1, \ldots, L(7)
\end{array}
$$

where $q_{1}, \ldots, q_{K}$ and $q_{K+1}, \ldots, q_{K+L}$ are the virtual uplink powers corresponding to the SUs and PUs, respectively. It is shown in [7] that problem (7) yields beamformers identical to those of problem (5). The authors then propose an iterative algorithm, based on the uplink-downlink duality, to obtain the solution. A similar iterative approach for the conventional beamforming problem has been presented in [16]. In contrast to the techniques of [9] and [15], these iterative approaches do not require any use of convex optimization tools, and, therefore, are simpler to implement.

\section{Robust CR Beamforming Problem}

In practice, the perfect knowledge of the true channel covariance matrices, $\boldsymbol{R}_{k},(k=1, \ldots, K)$ and $\boldsymbol{R}_{K+l}, \quad(l=$ $1, \ldots, L)$, is not available at the $\mathrm{SN}$ base station and there exists a mismatch between the true and the estimated channel covariance matrices. In order to take the CSI mismatch into account, we model the true channel covariance matrices as $\overline{\boldsymbol{R}}_{k} \triangleq \hat{\boldsymbol{R}}_{k}+\boldsymbol{\Delta}_{k}(k=1, \ldots, K)$ and $\overline{\boldsymbol{R}}_{K+l} \triangleq \hat{\boldsymbol{R}}_{K+l}+\boldsymbol{\Delta}_{K+l}$ $(l=1, \ldots, L)$ where $\hat{\boldsymbol{R}}_{k}$ and $\hat{\boldsymbol{R}}_{K+l}$ are the available estimates of the true channel covariance matrices of the $k$ th SU and $l$ th PU, respectively, and $\Delta_{k}$ and $\Delta_{K+l}$ contain the errors in these estimates. We assume that the Frobenius norm of these error matrices is upper-bounded by some known constants, i.e., $\left\|\boldsymbol{\Delta}_{k}\right\| \leq \alpha_{k}$ and $\left\|\boldsymbol{\Delta}_{K+l}\right\| \leq \beta_{l}$. In case of a channel mismatch, the solution of (5), obtained for the assumed covariances $\hat{\boldsymbol{R}}_{m}$ 
( $m=1, \ldots, K+L)$, generally violates at least part of the SU QoS and PU interference constraints for the true covariances $\boldsymbol{R}_{m},(m=1, \ldots, K+L)$. Using the introduced covariance error model, the robust $\mathrm{CR}$ beamforming problem corresponding to the problem (5) can be formulated as [11]

$$
\begin{aligned}
& \min _{\substack{\left\{, p_{1}, \ldots, p_{K}\right\} \\
\text { s.t. }}} \sum_{\substack{\left\|\boldsymbol{\Delta}_{k}\right\| \leq \alpha_{k} \\
\hat{\boldsymbol{R}}_{k}+\boldsymbol{\Delta}_{k} \geq 0}}^{K} \frac{p_{k} \boldsymbol{u}_{k}^{H}\left(\hat{\boldsymbol{R}}_{k}+\boldsymbol{\Delta}_{k}\right) \boldsymbol{u}_{k}}{\gamma_{k} \sum_{\substack{i=1 \\
i \neq k}}^{K} p_{i} \boldsymbol{u}_{i}^{H}\left(\hat{\boldsymbol{R}}_{k}+\boldsymbol{\Delta}_{k}\right) \boldsymbol{u}_{i}+\eta_{k}} \geq 1 ; k=1, \ldots, K(8) \\
& -\max _{\substack{\left\|\boldsymbol{\Delta}_{K+l} l\right\| \leq \beta_{l} \\
\hat{\boldsymbol{R}}_{K+l}+\boldsymbol{\Delta}_{K+l} \geq 0}} \gamma_{K+l} \sum_{i=1}^{K} p_{i} \boldsymbol{u}_{i}^{H}\left(\hat{\boldsymbol{R}}_{K+l}+\boldsymbol{\Delta}_{K+l}\right) \boldsymbol{u}_{i} \geq-1 ; l=1, \ldots, L
\end{aligned}
$$

where we have taken into account that the true covariances must be positive-semidefinite.

Based on the SDR approach, a solution to the robust problem (8) has been derived in [9] and [16] for the CR and the conventional beamforming cases, respectively. However, as pointed out in [11], the robust approaches of [9] and [16] use several conservative approximations that can affect the performance of the beamformers adversely. In [11], the authors convert the robust problem (8) to an equivalent form

$$
\begin{array}{ll} 
& \min _{\left\{\boldsymbol{U}, p_{1}, \ldots, p_{K}\right\}} \sum_{i=1}^{K} p_{k} \\
\text { s.t. } & -\operatorname{Tr}\left\{\hat{\boldsymbol{R}}_{k} \boldsymbol{A}_{k}\right\} \geq \eta_{k}+\alpha_{k}\left\|\boldsymbol{A}_{k}\right\| ; k=1, \ldots, K \\
& -\gamma_{K+l} \operatorname{Tr}\left\{\boldsymbol{C} \hat{\boldsymbol{R}}_{K+l}\right\} \geq \gamma_{K+l} \beta_{l}\|\boldsymbol{C}\|-1 ; l=1, \ldots, L
\end{array}
$$

where

$$
\boldsymbol{A}_{k} \triangleq \gamma_{k} \sum_{\substack{i=1 \\ i \neq k}}^{K} p_{i} \boldsymbol{u}_{i} \boldsymbol{u}_{i}^{H}-p_{k} \boldsymbol{u}_{k} \boldsymbol{u}_{k}^{H} ; \quad \boldsymbol{C} \triangleq \sum_{k=1}^{K} p_{k} \boldsymbol{u}_{k} \boldsymbol{u}_{k}^{H} .
$$

Next, defining $\boldsymbol{W}_{k} \triangleq p_{k} \boldsymbol{u}_{k} \boldsymbol{u}_{k}^{H}$, the problem (9) is reformulted as

$$
\begin{array}{ll} 
& \min _{\left\{\boldsymbol{W}_{i}\right\}} \sum_{i=1}^{K} \operatorname{Tr}\left\{\boldsymbol{W}_{i}\right\} \\
\text { s.t. } & -\operatorname{Tr}\left\{\hat{\boldsymbol{R}}_{k} \boldsymbol{A}_{k}\right\} \geq \eta_{k}+\alpha_{k}\left\|\boldsymbol{A}_{k}\right\| \\
& \boldsymbol{W}_{k} \geq 0, \operatorname{rank}\left(\boldsymbol{W}_{k}\right)=1 ; k=1, \ldots, K \\
& -\gamma_{K+l} \operatorname{Tr}\left\{\boldsymbol{C} \hat{\boldsymbol{R}}_{K+l}\right\} \geq \gamma_{K+l} \beta_{l}\|\boldsymbol{C}(t)\|-1 ; l=1, \ldots, L .
\end{array}
$$

Using SDR approach, the non-convex rank-one constraint is dropped to convert the problem (11) into a convex form.

Although the technique of [11] does not involve conservative approximations, it requires the use of interior point solvers. A difficulty arising in the context of SDR is that under particular channel conditions the matrices $\boldsymbol{W}_{k}$ solving (11) exhibit a rank larger than one [14]. Randomization techniques usually applied to obtain a rank-one solutions from a higher rank solution $\boldsymbol{W}_{k}$ can not be applied to the problem under consideration as these generally yield to infeasible points and, therefore, obtaining a rank-one solution from the corresponding higher rank solutions may not generally be possible. To overcome these difficulities, we develop an iterative robust
CR beamforming approach that uses the results from [11] and incorporates these with the iterative approach of [7]. The proposed technique enjoys simple implementation and does not require SDR.

\section{The Proposed Robust CR Beamforming Techniques}

Comparing the robust problem (9) with the non-robust problem (5), we observe that the terms $\alpha_{k}\left\|\boldsymbol{A}_{k}\right\|$ and $\gamma_{K+l} \beta_{l}\|\boldsymbol{C}\|$ are added to the QoS and PU interference constraints, respectively, in the robust case. These terms can be viewed as the penalty for adding the robustness to the non-robust problem (5). In the following we consider an iterative algorithm in which in each iteration the penalty terms $\left\|\boldsymbol{A}_{k}\right\|$ and $\|\boldsymbol{C}\|$ are assumed to be known from the previous iteration. Let $\boldsymbol{A}_{k}(t)$ and $\boldsymbol{C}(t)$ denote the matrices in (10) obtained in the $t$ th iteration then in each iteration we have the modified problem

$$
\begin{array}{ll} 
& \min \sum_{\left\{\boldsymbol{U}(t), p_{1}(t), \ldots, p_{K}(t)\right\}} \sum_{i=1}^{K} p_{k}(t) \\
\text { s.t. } & -\operatorname{Tr}\left\{\hat{\boldsymbol{R}}_{k} \boldsymbol{A}_{k}(t)\right\} \geq \hat{\eta}_{k}(t-1) ; k=1, \ldots, K \\
& -\gamma_{K+l} \operatorname{Tr}\left\{\boldsymbol{C} \hat{\boldsymbol{R}}_{K+l}\right\} \geq-\hat{\eta}_{K+l}(t-1) ; l=1, \ldots, L
\end{array}
$$

where

$$
\hat{\eta}_{k}(t) \triangleq \eta_{k}+\alpha_{k}\left\|\boldsymbol{A}_{k}(t)\right\|
$$

and

$$
\hat{\eta}_{K+l}(t) \triangleq 1-\gamma_{K+l} \beta_{l}\|\boldsymbol{C}(t)\| .
$$

From (12), we observe that when the penalty terms in the $t$ th iteration are fixed at $\left\|\boldsymbol{A}_{k}(t-1)\right\|$ and $\|\boldsymbol{C}(t-1)\|$, the robustness to the channel mismatch in the $\mathrm{CR}$ beamforming design is achieved by increasing the targets $\hat{\eta}_{m}(t)(m=1, \ldots, K+L)$ according to (13) and (14). Note, that for fixed $\left\|\boldsymbol{A}_{k}(t-1)\right\|$ and $\|\boldsymbol{C}(t-1)\|$, problem (12) is mathematically equivalent to (5). The resulting iterative algorithm can be described as

Algorithm 1: Robust CR beamforming using SDP solver

Initialization: Initialize $\boldsymbol{A}_{k}(0)=0$ and $\hat{\eta}_{k}(0)=\eta_{k}$, for $k=$ $1, \ldots, K, \boldsymbol{C}(0)=0$, and $\hat{\eta}_{K+l}(0)=1(l=1, \ldots, L)$.

Iterative loop: For $t=1,2, \ldots$ until convergence, iterate the following steps:

1) Beamformer computation: Solve the problem (12) using some SDP solver to compute $p_{k}(t)$ and $\boldsymbol{u}_{k}(t)$ for $k=1, \ldots, K$. 2) Targets update: Update $\hat{\eta}_{k}(t)$ using (13) and $\hat{\eta}_{K+l}(t)$ as

$$
\hat{\eta}_{K+l}(t)= \begin{cases}1-\gamma_{K+l} \beta_{l}\|\boldsymbol{C}(t)\|, & \text { if } 1-\gamma_{\mathrm{K}+1} \beta_{1}\|\boldsymbol{C}(\mathrm{t})\|>0 \\ 1, & \text { otherwise. }\end{cases}
$$

for $l=1, \ldots, L$.

Convergence check: For convergence, check the following condition:

$$
\left|\sum_{i=1}^{K} p_{k}(t)-\sum_{i=1}^{K} p_{k}(t-1)\right| \leq \delta
$$

where $\delta$ is a small positive constant.

Although, the above algorithm leads to a non-conservative rank-one solution of (8), it will have a relatively high complexity as we require to solve an SDP problem in each iteration. 
Simulation results show, that the algorithm generally converges after 4 to 6 iterations, but the number of iterations can go to over 100 for higher values of SINR required by the SUs. We propose to use the iterative fixed-point algorithm in [7] for solving the Step 1. In this case, Step 1 will consist of an inner loop to solve the beamformers. We propose to modify it by removing the outer iteration loop and updating the terms $\hat{\eta}_{k}(t)$ and $\hat{\eta}_{K+l}(t)$ in each iteration.

In the following we present the modified algorithm to solve the robust CR downlink beamforming problem (8). First, let us define $\boldsymbol{q} \triangleq\left[q_{1}, \ldots, q_{K+L}\right]^{T}$ and $\boldsymbol{p} \triangleq\left[\boldsymbol{p}_{1}^{T}, \boldsymbol{p}_{2}^{T}\right]^{T}$ where $\boldsymbol{p}_{1} \triangleq\left[p_{1}, \ldots, p_{K}\right]^{T}$ and $\boldsymbol{p}_{2} \triangleq\left[p_{K+1}, \ldots, p_{K+L}\right]^{T}$ are the vectors containing the downlink powers and the virtual downlink powers of the $K$ SUs and the $L$ PUs, respectively. We also introduce

$$
\begin{aligned}
& {[\boldsymbol{D}(t)]_{k, k} \triangleq \boldsymbol{u}_{k}(t)^{H} \hat{\boldsymbol{R}}_{k} \boldsymbol{u}_{k}(t)} \\
& {\left[\boldsymbol{G}_{2}(t)\right]_{l, j} \triangleq \frac{\gamma_{K+l}}{\hat{\eta}_{K+l}(t)} \boldsymbol{u}_{j}(t)^{H} \hat{\boldsymbol{R}}_{K+l} \boldsymbol{u}_{j}(t)} \\
& {\left[\boldsymbol{G}_{1}(t)\right]_{i, j} \triangleq \begin{cases}0, & i=j \\
\gamma_{i} \boldsymbol{u}_{j}(t)^{H} \hat{\boldsymbol{R}}_{i} \boldsymbol{u}_{j}(t), & i \neq j\end{cases} } \\
& \boldsymbol{\eta}(t) \triangleq\left[\hat{\eta}_{1}(t), \ldots, \hat{\eta}_{K}(t)\right]^{T}
\end{aligned}
$$

for all $l=1, \ldots, L$ and $k, i, j=1, \ldots, K$. The algorithm can then be described as

Algorithm 2: Robust iterative CR beamforming using uplinkdownlink duality

Initialization: Initialize $\boldsymbol{A}_{k}(0)=0, \hat{\eta}_{k}(0)=\eta_{k}$, and $q_{k}(0)=1$, for $k=1, \ldots, K, \hat{\eta}_{K+l}(0)=1$ and $q_{K+l}(0)=10^{-2}$ (can be chosen arbitrarily), for $l=1, \ldots, L$, and $\boldsymbol{C}(0)=0$.

Iterative loop: For $t=1,2, \ldots$ until convergence, iterate the following steps:

1) Beamformer update: Compute

$$
\mu_{k}(t)=\max _{\left\|\boldsymbol{u}_{k}(t)\right\|=1} \frac{q_{k}(t-1) \boldsymbol{u}_{k}(t)^{H} \hat{\boldsymbol{R}}_{k} \boldsymbol{u}_{k}(t)}{\boldsymbol{u}_{k}(t)^{H}(\boldsymbol{Q}+\boldsymbol{I}) \boldsymbol{u}_{k}(t)}
$$

where

$$
\boldsymbol{Q} \triangleq \sum_{i=1, i \neq k}^{K} q_{i}(t-1) \gamma_{i} \hat{\boldsymbol{R}}_{k}+\sum_{l=1}^{L} \frac{q_{K+l}(t-1) \gamma_{K+l}}{\hat{\eta}_{K+l}(t-1)} \hat{\boldsymbol{R}}_{k}
$$

and the vector $\boldsymbol{u}_{k}(t)$, for $k=1, \ldots, K$, from the solution of the corresponding generalized eigenproblem.

2) Transformation to the downlink domain: For the beamforming vectors obtained in step $\mathbf{1}$ compute the equivalent virtual downlink powers $\boldsymbol{p}_{1}(t)=\left(\boldsymbol{D}(t)-\boldsymbol{G}_{1}(t)\right)^{-1} \boldsymbol{\eta}$ and $\boldsymbol{p}_{2}(t)=$ $\boldsymbol{G}_{2}(t) \boldsymbol{p}_{1}(t)$ with $\boldsymbol{D}(t), \boldsymbol{G}_{1}(t), \boldsymbol{G}_{2}(t)$ and $\boldsymbol{\eta}(t)$ defined in (17)-(20). 3) Virtual uplink power allocation update (PUs): Compute

$$
q_{K+l}(t)= \begin{cases}p_{K+l}(t) q_{K+l}(t-1), & \text { if } \min \left\{p_{l}, \ldots, p_{K}\right\} \geq 0 \\ q_{K+l}(t-1), & \text { otherwise }\end{cases}
$$

for $l=1, \ldots, L$.

4) Virtual uplink power allocation update (SUs): Compute

$$
\boldsymbol{q}_{k}(t)=\frac{\boldsymbol{u}_{k}(t)^{H}(\hat{\boldsymbol{Q}}+\boldsymbol{I}) \boldsymbol{u}_{k}(t)}{\boldsymbol{u}_{k}(t)^{H} \hat{\boldsymbol{R}}_{k} \boldsymbol{u}_{k}(t)}
$$

where

$$
\hat{\boldsymbol{Q}} \triangleq \sum_{i=1, i \neq k}^{K} q_{i}(t-1) \gamma_{i} \hat{\boldsymbol{R}}_{k}+\sum_{l=1}^{L} \frac{q_{K+l}(t) \gamma_{K+l}}{\hat{\eta}_{K+l}(t-1)} \hat{\boldsymbol{R}}_{k}
$$

for $k=1, \ldots, K$.

5) Targets update: Compute $\hat{\eta}_{k}(t)$ and $\hat{\eta}_{K+l}(t)$ using (13) and (15), respectively.

Convergence check: For convergence, check the following conditions:

$$
\begin{aligned}
& \|\boldsymbol{q}(t)-\boldsymbol{q}(t-1)\| \leq \delta_{1} ; \quad p_{K+l}(t)-1 \leq \delta_{2} \\
& \left|\sum_{i=1}^{K} p_{k}(t)-\sum_{i=1}^{K} p_{k}(t-1)\right| \leq \delta_{3}
\end{aligned}
$$

for $l=1, \ldots, L$ where $\delta_{m}(m=1,2,3)$ are small positive constants.

We remark that if we remove the Step 5, i.e. keeping $\hat{\eta}_{k}(t)=\eta_{k}(t)(k=1, \ldots, K)$ and $\hat{\eta}_{K+l}(t)=1(l=1, \ldots, L)$, the Algorithm 2 reduces to the non-robust iterative algorithm of [7].

\section{Simulation Results}

We next present the simulation results for our proposed technique. We compare the proposed algorithm to the robust method of [11]. The base station is equipped with a uniform linear array of $N=7$ sensors spaced half a wavelength apart from each other, with $K=4$ SUs and $L=4$ PUs users. The SUs are located at $5^{\circ}, 15^{\circ}, 20^{\circ}$, and $40^{\circ}$, while the PUs are located at $10^{\circ}, 20^{\circ}, 25^{\circ}$, and $60^{\circ}$ relative to the array broadside. The users are assumed to be surrounded by a large number of local scatterers corresponding to a spread angle of $\sigma_{\theta}=3^{\circ}$ for both the SUs and the PUs, as seen from the base station. The channel covariance matrices, $\overline{\boldsymbol{R}}_{k}$ and $\overline{\boldsymbol{R}}_{K+l}$, are generated using the same model as in [15]. The user noises are assumed to be additive white Gaussian with variances $\sigma_{k}^{2}=1, k=1, \ldots, K$.

The channel error matrices, $\boldsymbol{\Delta}_{k}$ and $\boldsymbol{\Delta}_{K+l}$ are uniformly randomly generated in a sphere centered at zero with the radii $\alpha_{k}$ and $\beta_{l}$, respectively. The resulting error matrices are then added to $\overline{\boldsymbol{R}}_{k}$ and $\overline{\boldsymbol{R}}_{K+l}$, to obtain the estimated channel covariance matrices $\hat{\boldsymbol{R}}_{k}$ and $\hat{\boldsymbol{R}}_{K+l}$. If $\hat{\boldsymbol{R}}_{k}$ or $\hat{\boldsymbol{R}}_{K+l}$ have some negative eigenvalues, then the corresponding eigenvalues are replaced by 0 . We assume that $\gamma_{k}=\gamma, \alpha_{k}=\alpha, \beta_{l}=\beta$ and $\frac{1}{\gamma_{K+l}}=\frac{1}{\gamma}=5 \mathrm{~dB}$, for all $k=1, \ldots, K$ and $l=1, \ldots, L$. A total number of 300 Monte-Carlo runs has been used.

In Fig. 1, we compare the percentage of feasible cases versus the SINR required by the SUs, for the proposed technique and the approach in [11], for different values of $\alpha$ and $\beta$. A beamforming solution is considered as feasible, if it satisfies all the constraints in problem (5) for the true covariance matrices. The technique in [11] shows similar feasibility for both values of $\alpha$ and $\beta$, while the feasibility of the proposed technique decreases for higher values of the required SINR. Next, in Fig. 2, we plot the transmitted power for the two techniques. Here, we consider only those cases when both approaches are feasible. Both schemes show similar performance and the transmitted power for both methods increases with the increase in the SINR required by the SUs. For higher values 


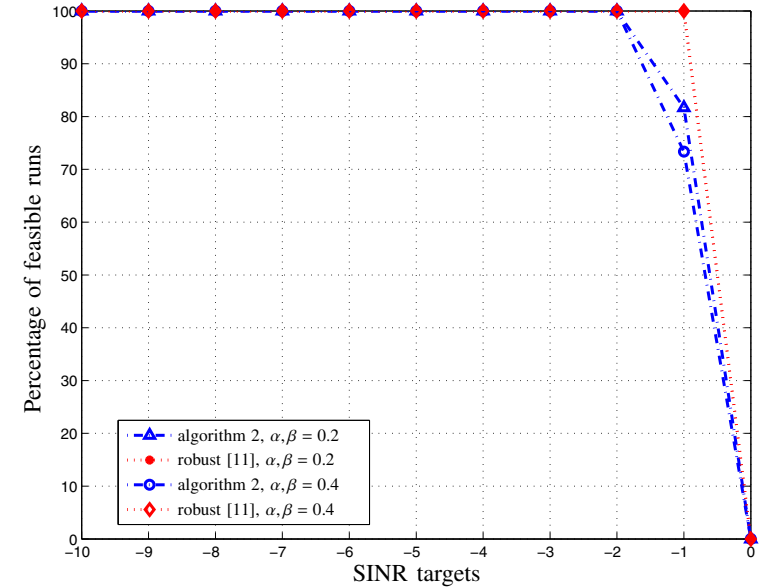

Fig. 1. Feasibility percentage of different schemes.

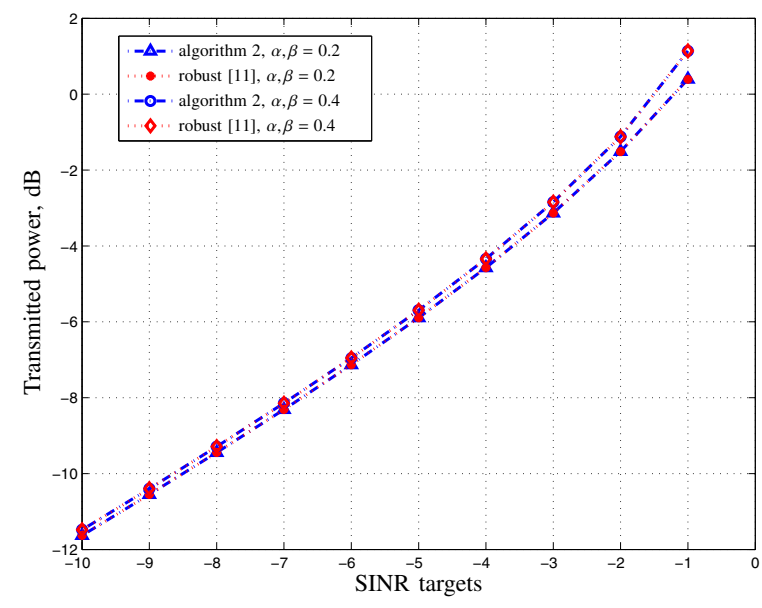

Fig. 2. Total transmitted power versus SINR required by SUs.

of $\alpha$ and $\beta$, both approaches require higher transmitted power, as expected. Finally, in Fig. 3, we plot the number of iterations required by the propsed technique to converge to the optimal solution. We can see that the number of iterations remains below 20 for most of the SINR values. For higher SINR values, however, the number of iterations grows significantly. Simulations results verify that the proposed technique provides results comparable with those of the technique in [11], while enjoying a much simpler and practical implementation, and guaranteeing a rank-one solution.

\section{REFERENCES}

[1] "Report of the Spectrum Efciency Working Group," FCC Spectrum Policy Task Force, 2002 [Online]. Available: http://www.fcc.gov/sptf/files/SEWGFinalReport 1.pdf

[2] S. Haykin, "Cognitive radio: Brain-empowere $\bar{d}$ wireless communications," IEEE J. Sel. Areas in Communications, vol. 23, no. 2, pp. 201220, Feb. 2005.

[3] G. Scutari and D. P. Palomar, "MIMO cognitive radio: A game theoretical approach," IEEE Trans. Sig. Processing, vol. 58, no. 2, pp. 761-780, Feb. 2010.

[4] J. Mitola and G. Q. Maguire, "Cognitive radio: Making software radios more personal," IEEE Personal Communications, vol. 6, no. 6, pp. 1318, Aug. 1999.

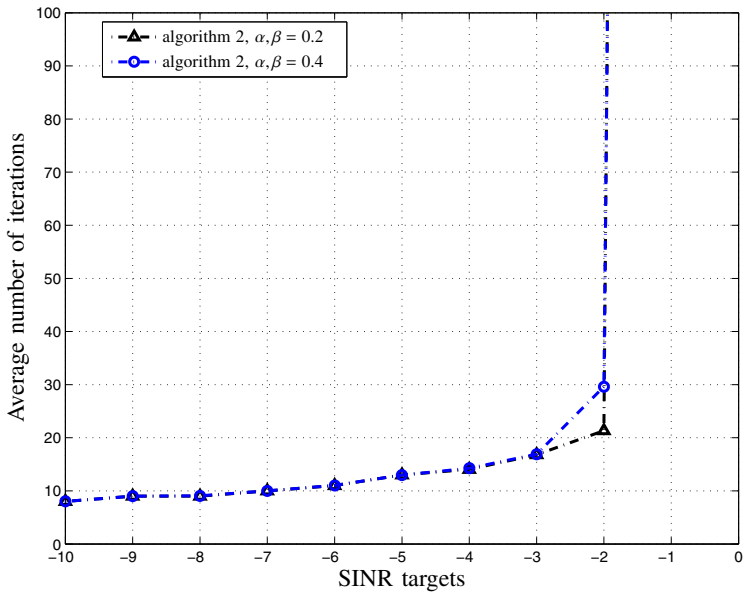

Fig. 3. Number of iterations versus SINR required by SUs for the proposed algorithm

[5] D. Calin, H. Claussen, and H. Uzunalioglu, "On femto deployment architectures and macrocell offloading benefits in joint macro-femto deployments," IEEE Commun. Magazine, vol. 48, no. 1, pp. 26-32, Jan. 2010

[6] L. Zhang, Y.-C. Liang, and Y. Xin, "Joint beamforming and power control for multiple access channels in cognitive radio networks," IEEE J. Sel. Areas in Communications, vol. 26, no. 1, pp. 38-51, Jan. 2008.

[7] M. Pesavento, D. Ciochina, and A. B. Gershman, "Iterative dual downlink beamforming for cognitive radio networks", in Proc. CROWNCOM'10, Cannes, France, June 2010.

[8] M. H. Islam, Y.-C. Liang, and A. T. Hoang, "Joint power control and beamforming for cognitive radio networks," IEEE Trans. Wireless Communications, vol. 7, no. 7, pp. 2415-2419, Jul. 2008.

[9] K. Cumanan, R. Krishna, V. Sharma, and S. Lambotharan, "Robust interference control techniques for multiuser cognitive radios using worstcase performance optimization," in Proc. 42nd Asilomar Conference on Signals, Systems and Computers, Oct. 2008, pp. 378-382.

[10] G. Zheng, K. K. Wong, and B. Ottersten, "Robust cognitive beamforming with bounded channel uncertainties," IEEE Trans. Sig. Processing, pp. 4871-4881, Dec. 2009.

[11] I. Wajid, M. Pesavento, Y. C. Eldar, and A. B. Gershman, "Robust downlink beamforming for cognitive radio networks," in Proc. GLOBECOM'10, Miami, Florida, USA, Dec. 2010.

[12] B. Hassibi and B. M. Hochwald, "How much training is needed in multiple-antenna wireless links?" IEEE Trans. Inf. Theory, vol. 49, no. 4, pp. 951-963, Apr. 2003.

[13] D. J. Love, R. W. Heath, W. Santipach, and M. L. Honig, "What is the value of limited feedback for MIMO channels?" IEEE Comm. Mag., vol. 42, pp. 54-59, Oct. 2004.

[14] I. Wajid, M. Pesavento, Y. C. Eldar, and A. B. Gershman, "Robust Downlink Beamforming using Second-Order Channel State Information for Conventional and Cognitive Radio Networks, "to be submitted to IEEE Trans. Sig. Processing.

[15] M. Bengtsson and B. Ottersten, "Optimal downlink beamforming using semidefinite optimization," in Proc. 37th Annual Allerton Conf. Communications, Control and Computing, Sep. 1999, pp. 987-996.

[16] M. Bengtsson and B. Ottersten, "Optimal and suboptimal transmit beamforming," in Handbook of Antennas in Wireless Communications, L. C. Godara, Ed. Boca Raton, FL: CRC Press, Aug. 2001, ch. 18 